\title{
Beta-blocker initiation and adherence after hospitalization for acute myocardial infarction.
}

\author{
Vittorio Maio, PharmD, MS, MSPH \\ Jefferson School of Population Health \\ Massimiliano Marino, PhD \\ 2. Clinical Governance, Regional Health Care Agency, Emilia-Romagna, Bologna, Italy \\ Mary Robeson, MD \\ Jefferson Medical College \\ Joshua J Gagne, PharmD, MS \\ Harvard School of Public Health
}

Follow this and additional works at: https://jdc.jefferson.edu/healthpolicyfaculty

Part of the Health Services Research Commons

Let us know how access to this document benefits you

\section{Recommended Citation}

Maio, PharmD, MS, MSPH, Vittorio; Marino, PhD, Massimiliano; Robeson, MD, Mary; and Gagne, PharmD, MS, Joshua J, "Beta-blocker initiation and adherence after hospitalization for acute myocardial infarction." (2011). College of Population Health Faculty Papers. Paper 47. https://jdc.jefferson.edu/healthpolicyfaculty/47

This Article is brought to you for free and open access by the Jefferson Digital Commons. The Jefferson Digital Commons is a service of Thomas Jefferson University's Center for Teaching and Learning (CTL). The Commons is a showcase for Jefferson books and journals, peer-reviewed scholarly publications, unique historical collections from the University archives, and teaching tools. The Jefferson Digital Commons allows researchers and interested readers anywhere in the world to learn about and keep up to date with Jefferson scholarship. This article has been accepted for inclusion in College of Population Health Faculty Papers by an authorized administrator of the Jefferson Digital Commons. For more information, please contact: JeffersonDigitalCommons@jefferson.edu. 


\author{
As submitted to: \\ European Journal of Cardiovascular Prevention and Rehabiliation \\ Volume 18, Issue 3, June 2011, Pages 438-445
}

And later published as:

\title{
Beta-blocker initiation and adherence after hospitalization for acute myocardial infarction
}

\section{DOI: $10.1177 / 1741826710389401$}

\section{Running title: POST-AMI BETA-BLOCKER USE}

Vittorio Maio, PharmD, MS, MSPH, ${ }^{1}$ Massimiliano Marino, $\mathrm{PhD},{ }^{2}$ Mary Robeson, $\mathrm{MS},{ }^{3} \mathrm{Joshua}$ J. Gagne, PharmD, $\mathrm{MS}^{4,5}$

1. School of Population Health, Thomas Jefferson University, Philadelphia, PA, USA

2. Clinical Governance, Regional Health Care Agency, Emilia-Romagna, Bologna, Italy

3. Center for Medical Education and Health Care Research, Jefferson Medical College, Philadelphia, PA, USA

4. Division of Pharmacoepidemiology and Pharmacoeconomics, Brigham and Women's Hospital and Harvard Medical School, Boston, MA, USA

5. Department of Epidemiology, Harvard School of Public Health, Boston, MA, USA

\author{
Corresponding author: \\ Vittorio Maio, PharmD, MS, MSPH \\ School of Population Health \\ Thomas Jefferson University \\ 1015 Walnut Street \\ Suite 115 \\ Philadelphia, PA 19107 \\ (T): 215-955-1821
}


(F): 215-923-7583

(E): vittorio.maio@jefferson.edu

\section{Abstract Word Count: 249 \\ Text Word Count: 2,950 \\ Number of References: 33 \\ Number of Figures: 2 \\ Number of Tables: 2 \\ ACKNOWLEDGEMENTS}

This research was conducted through a collaborative agreement between the Regional Health

Care Agency, Assessorato alla Sanità, Emilia-Romagna, Italy, and Jefferson Medical College, Philadelphia, PA, USA.

We thank Carol Rabinowitz and Emily Gavin from the Center for Research in Medical

Education and Health Care for assistance with data management and analytical support, and

Daniel Z. Louis, MS, from the Center for Research in Medical Education and Health Care and Roberto Grilli, MD, and Rossana De Palma, MD, from the Regional Health Care Agency, Regione Emilia-Romagna, Italy, for their input and guidance with the project. 


\section{INTRODUCTION}

Long-term use of beta-blockers after acute myocardial infarction (AMI) may reduce total mortality by as much as $26 \%$ [1]. Given the high incidence of AMI, it has been estimated that if all AMI survivors initiated and continued beta-blocker therapy for 20 years in the US alone, as many as 4,300 fewer deaths from chronic heart disease and 3,500 fewer repeat AMIs would occur in the United States [2].

Guidelines from the American College of Cardiology (ACC), the American Heart Association (AHA), and the European Society of Cardiology recommend use of beta-blockers in AMI patients without contraindications to such treatment [3-5]. However, post-AMI beta-blocker therapy is often underutilized [6-10]. Only $61 \%$ of patients who were candidates for post-AMI beta-blocker therapy actually initiated it in British Columbia, Canada, between 2001 and 2004 [9]. Furthermore, post-AMI beta-blockade is maximally effective only when patients adhere to the therapy and persist on it over the long-term [11]. However, observed adherence to and persistence with post-AMI beta-blocker therapy has also been low among those who initiate such therapy [12].

Little is known about recent patterns and predictors of post-AMI beta-blocker initiation [13] and recent trends in adherence to such therapy. Most studies currently available in the medical literature have examined patterns and predictors of beta-blocker therapy only up to 2004 and most of this evidence is from North America. Drug prescribing and utilization patterns can vary widely between countries and also within countries over time [14,15]. Thus, we sought to: (1) estimate the proportion of patients without contraindications who initiated beta-blocker therapy 
after AMI in Regione Emilia-Romagna (RER), Italy; (2) examine predictors, including patient and clinical characteristics and use of alternative medications, of beta-blocker initiation after AMI; and (3) estimate patient adherence to beta-blocker therapy after AMI using data from 2003 to 2008 .

\section{METHODS}

\section{Data source}

We used the healthcare database from RER, comprising all of the approximately 4 million RER residents over the period 2003 to 2008 . This database captures health service utilization information for the entire region, including hospital discharge abstract data, such as diagnosis and procedure codes, and outpatient prescription drug dispensing data. Individuals can be linked across data files with anonymous patient identifiers and can be followed longitudinally. This study complies with the Declaration of Helsinki and was approved by the Institutional Review Board of Thomas Jefferson University, Philadelphia, PA.

\section{Cohort identification}

We identified a cohort of patients with AMI comprising all RER residents aged 35 and older discharged alive from a regional hospital between January 1, 2004 and December 31, 2007 with a primary diagnosis of AMI (ICD-9 codes 410.x1) [16]. The index AMI admission for each patient was defined as the first AMI admission identified in the study period. We excluded patients discharged alive but whose length of stay for the index AMI admission was less than 3 days, as recorded AMI codes may have been for rule-out purposes in these cases [16,17]. 
Patients were also excluded if they died within 30 days of discharge to ensure a minimum time window for measuring beta-blockers use in the outpatient setting.

Patients were also excluded if they had absolute or relative contraindications to beta-blockers. Specifically, based on primary and secondary inpatient diagnoses in the year prior to index hospitalization, we excluded those with hypotension, heart block $>1$ degree, sinus bradycardia, chronic obstructive pulmonary disease, or peripheral vascular disease. Patients with asthma were also excluded and were identified as those with a prescription record for inhaled corticosteroids in the 6 months prior to the index hospitalization or those with a primary or secondary hospital discharge diagnosis of asthma in year prior to the index AMI admission. Patients with type 1 diabetes mellitus, defined as those who had received insulin in the 6 months prior to or 3 months following the index AMI admission, were also excluded [17].

\section{Covariates}

Potential predictors of beta-blocker initiation were identified from inpatient data and prescription drug dispensing data in the year preceding index hospitalization - as early as the beginning of 2003 (a detailed list of ICD-9-CM codes and of Anatomical Therapeutic Chemical (ATC) classification system codes are available from the authors). We retrieved demographic information, including age, gender, and geographic location. We also collected information on cardiovascular health services use, including hospital admissions with primary diagnoses of angina, ischemic heart disease, congestive heart failure, and other cardiovascular diseases, as well as use of specific medications for cardiovascular conditions, including digoxin, angiotensinconverting enzyme (ACE) inhibitors, nitrates, beta-blockers, calcium channel blockers, and 
angiotensin II receptor blockers (ARBs) [18]. Comorbidities were ascertained from non-cardiac hospital admissions with primary diagnoses for other conditions that may increase mortality risk [18]. We also used the Chronic Condition Drug Group (CCDG), a prescription drug-based comorbidity index [19]. CCDGs were developed in the same RER database and have been applied in other published studies $[14,15,20]$. Also, we computed the number of different drug products filled in the 6 months before hospitalization as a proxy for health status [21]. Finally, we constructed indicator variables for intensity of the in-hospital stay, including length of stay, receipt of invasive cardiac procedures, and whether the patient received specialized care defined as spending at least part of their stay in the cardiac surgery, intensive care, cardiology, or critical care units.

\section{Outcome measures and statistical analysis}

We estimated the proportion of patients in the eligible cohort with at least one record of a dispensing for a beta-blocker within 90 days after discharge from the index hospitalization. We conducted a logistic regression analysis to identify which factors were independent predictors of beta-blocker initiation in this cohort. Dichotomous post-AMI beta-blocker use was the dependent variable in the model. All other covariates described above, including demographics, comorbid conditions, and cardiovascular health services use, were entered into the model simultaneously as independent variables to identify which factors were associated with betablocker use while controlling for all other factors in the model. Odds ratios (ORs) and their 95\% confidence intervals were estimated for the association between beta-blocker initiation and each of the independent variables. All analyses were conducted using SAS (version 9.1, Cary, NC). 
Among cohort members with at least one prescription for a post-AMI beta-blocker, we estimated the proportion of days covered (PDC), or medication possession ratio, for beta-blocker therapy at 6 and 12 months from the first beta-blocker prescription dispensed. This interval-based PDC is a commonly used measure of drug availability and is a function of both adherence to (i.e. patient takes prescribed medication according to schedule) and persistence with (i.e. patient continues to take therapy) drug therapy over a defined time interval [22,23]. To estimate the 6- and 12-month PDCs, we first computed the number of days covered by beta-blockers within each time period of interest as the total number of Defined Daily Doses (DDDs) for any type of beta-blocker prescribed to each individual [22,24]. The DDD is a technical unit of measurement, used by the World Health Organization, which reflects the average adult dose used for the main indication as reflected by the Anatomical Therapeutic Chemical (ATC) classification system code [25]. We then divided the number of days covered by beta-blockers by 180 and 360 for the 6- and 12month estimate, respectively, and multiplied by 100 to arrive at the estimated PDC. We excluded patients who died within 6 and 12 months after the index AMI, from each time interval, respectively. We used a priori PDC cutoffs of $\geq 80 \%, 50 \%$ to $<80 \%$, and $<50 \%$ to classify patients as adherent, moderately adherent, and non-adherent, respectively [6]. We also calculated the mean 6- and 12-month PDC among initiators stratified by year of hospitalization and performed a Cochran-Armitage test for trend over time in the proportion of initiators who were adherent.

\section{RESULTS}

\section{Study Cohort}


We identified 28,001 patients who met the cohort eligibility criteria. Of these, 3,634 (13\%) were excluded because they had one or more contraindications to beta-blockers. Thus, the final study cohort comprised 24,367 eligible patients hospitalized for AMI in RER between 2004 and 2007 (Table 1). Selected demographic and clinical information as well as factors related to the index hospitalization are reported in Table 1, and are fully reported in the online supplement. Of the eligible cohort, 16,383 (67\%) received at least one outpatient dispensing for a beta-blocker in the 90 days after discharge from the index hospitalization (Table 1). Propranolol (61\%) was the most commonly dispensed index beta-blocker, followed by bisoprolol (19\%), carvedilol (16\%), and atenolol (3\%).

Following a slight increase in proportion of beta-blocker initiators between 2004 and 2005, the proportion remained relatively unchanged between 2005 and 2007 (Figure 1). In general, those who initiated beta-blockers were younger and appeared healthier than those who did not. For example, beta-blocker initiators were hospitalized less often and had fewer comorbid conditions in the year preceding index hospitalization as compared to patients who did not initiate betablockers after discharge. Furthermore, those who initiated beta-blockers were more likely to have also received specialized inpatient care and were more likely to have undergone invasive cardiac procedures (Table 1 and online supplement).

\section{Predictors of beta-blocker use}

Table 2 presents the results of the multivariable logistic regression model examining independent predictors of post-AMI beta-blocker initiation (see online supplement for full results). Adjusted for other covariates in the model, the odds of initiating a post-AMI beta-blocker decreased 
monotonically with increasing age (Table 2). Undergoing coronary artery bypass graft (CABG) surgery (OR, 2.37; 95\% CI, 2.00-2.81), percutaneous transluminal coronary angioplasty (PTCA; OR, 1.42; 95\% CI, 1.31-1.54), and cardiac catheterization (OR, 1.14; 95\% CI, 1.11-1.32) remained strong predictors of initiating post-AMI beta-blocker therapy (Table 2). Use of certain types of drugs for cardiovascular conditions in the 6 months leading up to index hospitalization were also strong predictors of beta-blocker use after discharge, including use of ACE-inhibitors (OR, 1.19; 1.11-1.28), ARBs (OR, 1.15; 95\% CI, 1.05-1.26) and beta-blockers (OR, 1.81; 95\% CI, 1.67-1.96).

\section{Adherence to beta-blocker therapy}

Within the first 6 months after discharge, $513(3.1 \%)$ subjects who received post-AMI betablockers died and were excluded from both the 6-month PDC estimation. A total of 961 (5.9\%) of beta-blocker initiators died during the follow-up year and were excluded from the 12-month PDC estimation. Monotonic decreases in mean 6- and 12-month PDC estimates were observed between 2004 and 2007. Mean 6-month PDC was 64.5\% in 2004, 62.2\% in 2005, 60.3\% in 2006, and 58.4\% in 2007. Mean 12-month PDC was 59.3\% in 2004, 57.3\% in 2005, 55.2\% in 2006, and 53.8\% in 2007. The proportion of beta-blocker users who were adherent at 6 months following therapy initiation and at 12 months following initiation (Figure 2) also decreased over time ( $p$ for trend $<0.001$ for both 6- and 12-month PDC).

\section{DISCUSSION}

Among patients identified as being eligible for beta-blocker treatment following AMI hospitalization in RER, only two-thirds initiated such therapy between 2004 and 2007, despite 
compelling evidence of substantial reductions in morbidity and mortality associated with such treatment. This proportion is within the range of estimates previously observed in other countries. For example, in Canada, $61 \%$ of patients initiated post-AMI beta-blocker therapy in British Columbia, between 2001 and 2004 [9] and 77\% were initiators in Ontario over the same period [6]. The $67 \%$ that we observed was a substantial improvement over the $9 \%$ to $31 \%$ uptake observed by Avanzini and colleagues in Italy in 1983-1994 [13].

Adherence to and persistence with beta-blocker therapy in the 6 and 12 months following treatment initiation was low, and much lower than that observed in other studies $[6,12,26]$. We also observed that the mean PDC and the proportion of patients classified as adherent decreased over time, even though the proportion of patients surviving AMI hospitalization who initiated beta-blocker therapy was largely unchanged during this period. These findings are in contrast with observations from the US and Canada which indicate that adherence with beta-blocker therapy was on the rise between the 1990s and 2003 [12,26]. A particular strength of our study is that it uses recent data. Further investigation in other regions and countries using recent data is required to understand if our observed decrease in adherence is specific to RER, or to Italy, or if trends in other countries also regressed after 2003.

Several demographic variables, frailty and comorbidity indicators, and factors related to index hospitalization were identified as independent predictors of post-AMI beta-blocker use. Consistent with a similar population-based study in Finland [27], we found that beta-blocker initiation was inversely associated with age. In general, older individuals and those with conditions indicating illness and frailty were less likely to initiate beta-blockers after AMI. It is 
possible that prescribing of drugs for secondary prevention may often be avoided in patients with poor prognoses, based on the belief that preventive strategies may confer little benefit while adding the burden of an additional treatment in these patients. Providers may also be reluctant to prescribe beta-blockers to older patients because of concern of adverse events.

An important limitation of our study is that we were unable to distinguish between patients who did not receive a beta-blocker prescription from a provider following AMI hospitalization and those that did receive a prescription but never filled it (so-called primary non-adherence) [28]. Among post-AMI patients in other countries, it has been estimated that up to 1 in 5 patients exhibit primary non-adherence [28,29]. Thus, a substantial proportion of the $33 \%$ of patients that we identified as non-initiators may be patients who received a beta-blocker prescription but did not fill it. Whether due to suboptimal prescribing or to primary non-adherence, it appears that many patients in RER who could benefit from beta-blocker therapy are not using it. Indeed, results from several studies suggest that older patients benefit considerably from beta-blockade $[18,30]$. Other studies have also found that the patients who are least likely to receive post-AMI beta-blocker therapy may be those in whom beta-blockade confers the greatest benefit [8].

We found that patients who underwent invasive cardiac procedures during hospitalization for AMI (i.e. CABG, PTCA, and cardiac catheterization) were more likely to initiate beta-blocker therapy after discharge as compared to those patients who did not undergo such procedures. Consistent with prescribing decisions based on prognosis, this observation may reflect the notion that those who receive invasive procedure are those expected to have better prognoses and those that are expected to have better prognoses are those who are more likely to receive post- 
discharge beta-blocker therapy. Alternatively, this finding may suggest that hospitals with the capacity and expertise to provide invasive cardiac treatment for AMI are also more likely to provide appropriate medical management, such as post-AMI beta-blocker treatment. On a patient-level, this finding could reflect the notion that patients who consent to invasive procedures are more likely to initiate subsequent preventive health measures. Regardless of the explanation, this finding is in stark contrast with results from other studies. One US-based study found that patients receiving intensive in-hospital treatment, measured as the receipt of cardiac catheterization within 30 days of AMI, were less likely to receive post-AMI beta-blocker therapy [31]. In another study among US Medicare patients with AMI, geographic regions where invasive procedures are more common were less likely to provide beta-blocker therapy to their patients [32]. Additional research in RER should focus on the relation between post-AMI betablocker use and geographic provision of intensive cardiac care in the region (i.e. hub and spoke [33]).

Given the solid scientific foundation for post-AMI beta-blockade and the low concordance with such evidence-based care that we observed in routine practice, health policy actions may be warranted. Quality improvement initiatives, such as pay-per-performance programs, could be established to encourage appropriate beta-blocker prescribing for eligible patients in accordance with guidelines. In addition, educational programs targeting AMI survivors could improve patient adherence to appropriate beta-blocker therapy. Such patient-directed activities could focus on those individuals who are least likely to receive appropriate care but in whom the benefits of such care confer the greatest benefit, such as older individuals. 
Our study has several additional limitations. First, it is not possible to accurately identify those patients in whom beta-blockers are ineffective or not tolerated using administrative claims data and sufficient information to identify all patients with contraindications to therapy may be limited. For example, we could not identify patients who may have received beta-blockers in the hospital, were intolerant, and subsequently discontinued therapy prior to discharge.

Furthermore, we relied on definitions of contraindications used by previous investigators and we were unable to assess their validity in the RER database. Thus, due to misclassification, we may have included in our cohort patients with relative contraindications that are not captured in administrative healthcare data. Also, the regional policy of directly distributing medications to patients from hospital pharmacies both at discharge and for outpatient treatment could have resulted in a slight underestimate of post-AMI beta-blocker use since these data are not captured at an individual level in the RER database. Finally, we used DDDs to estimate a proxy of days supply in order to derive the PDC. However, DDDs are fixed units of measurement and may not always reflect prescribed daily doses for individual patients [25]. As a result, DDDs provide only a rough estimate of utilization. It is possible that some PDC misclassification occurred, but this is not likely to have substantially affected the results.

In conclusion, despite the well-documented benefits of beta-blockade after AMI, beta-blocker therapy remained underutilized in RER. Utilization was less likely among older individuals and among those with indicators of frailty, such as multiple and various comorbid conditions, even though these patients may benefit from post-AMI beta-blockade. Among those who did initiate post-AMI beta-blocker treatment, adherence to and persistence with such therapy was also low and decreased over time in RER between 2004 and 2007. 


\section{REFERENCES}

1. Lau J, Antman EM, Jimenez-Silva J, Kupelnick B, Mosteller F, Chalmers TC. Cumulative meta-analysis of therapeutic trials for myocardial infarction. N Engl J Med 1992; 327:248254.

2. Phillips KA, Shlipak MG, Coxson P, Heidenreich PA, Hunink MG, Goldman PA, Williams LW, Weinstein MC, Goldman L. Health and economic benefits of increased beta-blocker use following myocardial infarction. JAMA 2000; 284:2748-2754.

3. Ryan TJ, Antman EM, Brooks NH, Califf RM, Hillis LD, Hiratzka LF, Rapaport E, Riegel B, Russell RO, Smith EE 3rd, Weaver WD, Gibbons RJ, Alpert JS, Eagle KA, Gardner TJ, Garson A Jr, Gregoratos G, Ryan TJ, Smith SC Jr. 1999 update: ACC/AHA guidelines for the management of patients with acute myocardial infarction: executive summary and recommendations: A report of the American College of Cardiology/American Heart Association Task Force on Practice Guidelines (Committee on Management of Acute Myocardial Infarction). Circulation 1999; 100:1016-1030.

4. López-Sendón J, Swedberg K, McMurray J, Tamargo J, Maggioni AP, Dargie H, Tendera M, Waagstein F, Kjekshus J, Lechat P, Torp-Pedersen C; Task Force On Beta-Blockers of the European Society of Cardiology. Expert consensus document on beta-adrenergic receptor blockers. Eur Heart J 2004; 25:1341-1362.

5. De Backer G, Ambrosioni E, Borch-Johnsen K, Brotons C, Cifkova R, Dallongeville J, Ebrahim S, Faergeman O, Graham I, Mancia G, Cats VM, Orth-Gomér K, Perk J, Pyörälä K, Rodicio JL, Sans S, Sansoy V, Sechtem U, Silber S, Thomsen T, Wood D; European Society of Cardiology Committee for Practice Guidelines. European guidelines on cardiovascular disease prevention in clinical practice: third joint task force of European and other societies 
on cardiovascular disease prevention in clinical practice (constituted by representatives of eight societies and by invited experts). Eur J Cardiovasc Prev Rehabil 2003; 10:S1-S10.

6. Rasmussen JN, Chong A, Alter DA. Relationship between adherence to evidence-based pharmacotherapy and long-term mortality after acute myocardial infarction. JAMA 2007; 297:177-186.

7. Krumholz HM, Radford MJ, Wang Y, Chen J, Heiat A, Marciniak TA. National use and effectiveness of beta-blockers for the treatment of elderly patients after acute myocardial infarction: National Cooperative Cardiovascular Project. JAMA 1998; 280:623-629.

8. Wei L, Flynn R, Murray GD, MacDonald TM. Use and adherence to beta-blockers for secondary prevention of myocardial infarction: who is not getting the treatment? Pharmacoepidemiol Drug Saf 2004; 13:761-766.

9. Schneeweiss S, Patrick AR, Maclure M, Dormuth CR, Glynn RJ. Adherence to beta-blocker therapy under drug cost-sharing in patients with and without acute myocardial infarction. Am J Manag Care 2007; 13:445-452.

10. Rochon PA, Anderson GM, Tu JV, Clark JP, Gurwitz JH, Szalai JP, Lau P. Use of betablocker therapy in older patients after acute myocardial infarction in Ontario. CMAJ 1999; 161:1403-1408.

11. Choudhtry NK, Winkelmayer WC. Medication adherence after myocardial infarction: a long way left to go. J Gen Inten Med 2008; 23:216-218.

12. Choudhry NK, Setoguchi S, Levin R, Winkelmayer WC, Shrank WH. Trends in adherence to secondary prevention medications in elderly post-myocardial infarction patients. Pharmacoepidemiol Drug Saf 2008; 17:1189-1196. 
13. Avanzini F, Zuanetti G, Latini R, Colombo F, Santoro E, Maggioni AP, Franzosi MG, Tognoni G. Use of beta-blocking agents in secondary prevention after myocardial infarction: A case for evidence-based medicine? GISSI experience, 1984-1993. The Gruppo Italiano di Studio sulla Sopravvivenza nell'Infarto Miocardico (GISSI) Investigators. Eur Heart J 1997; 18:1447-1456.

14. Gagne JJ, Maio V, Rabinowitz C. Prevalence and predictors of potential drug-drug interactions in Regione Emilia-Romagna, Italy. J Clin Pharm Ther 2008; 33:141-151.

15. Gagne JJ, Maio V, Berghella V, Louis DZ, Gonnella JS. Prescription drug use during pregnancy: a population-based study in Regione Emilia-Romagna, Italy. Eur J Clin Pharmacol 2008; 64:1125-32.

16. Kiyota Y, Schneeweiss S, Glynn RJ, Cannuscio CC, Avorn J, Solomon DH. The accuracy of Medicare claims-based diagnosis of acute myocardial infarction. Am Heart J 2004; 148:99104.

17. Iezzoni LI, Burnside S, Sickles L, Moskowitz MA, Sawitz E, Levine PA. Coding of acute myocardial infarction. Clinical and policy implications. Ann Intern Med 1988; 109:745-751.

18. Soumerai SB, McLaughlin TJ, Spiegelman D, Hertzmark E, Thibault G, Goldman L. Adverse outcomes of underuse of beta-blockers in elderly survivors of acute myocardial infarction. JAMA 1997; 277:115-121.

19. Maio V, Yuen E, Rabinowitz C, Louis D, Jimbo M, Donatini A, Mall S, Taroni F. Using pharmacy data to identify those with chronic conditions in Emilia Romagna, Italy. J Health Serv Res Policy 2005; 10:232-238. 
20. Maio V, Yuen EJ, Novielli K, Smith KD, Louis DZ. Potentially inappropriate medication prescribing for elderly outpatients in Emilia Romagna, Italy: A population-based cohort study. Drugs Aging 2006; 23:915-924.

21. Schneeweiss S, Wang PS, Avorn J, Maclure M, Levin R, Glynn RJ. Consistency of performance ranking of comorbidity adjustment scores in Canadian and U.S. utilization data. J Gen Intern Med 2004; 19:444-450.

22. Choudhry NK, Shrank WH, Levin RL, Lee JL, Jan SA, Brookhart MA, Solomon DH. Measuring concurrent adherence to multiple related medications. Am J Manag Care 2009; 15:457-64.

23. Andrade SE, Kahler KH, Frech F, Chan KA. Methods for evaluation of medication adherence and persistence using automated databases. Pharmacoepidemiol Drug Saf 2006; 15:565-74; discussion 575-7.

24. Atella V, Peracchi F, Depalo D, Rossetti C. Drug compliance, co-payment and health outcomes: Evidence from a panel of Italian patients. Health Econ 2006; 15:875-892.

25. ATC/DDD Index. WHO Collaborating Centre for Drug Statistics Methodology. 2006. Available at: http://www.whocc.no/atcddd. Accessed: October 11, 2007.

26. Lamb DA,Eurich DT, McAlister FA, Tsuyuki RT, Semchuk, Wilson TW, Blackburn DF. Changes in adherence to evidence-based medications in the first year after initial hospitalization for heart failure: observational cohort study from 1994 to 2003. Circ Cardiovasc Qual Outcomes 2009; 2:228-235.

27. Salomaa V, Pääkkönen R, Hämäläinen H, Niemi M, Klaukka T. Use of secondary preventive medications after the first attack of acute coronary syndrome. Eur J Cardiovasc Prev Rehabil 2007; 14:386-391. 
28. Jackevicius CA, Li P, Tu JV. Prevalence, predictors, and outcomes of primary nonadherence after acute myocardial infarction. Circulation 2008; 117:1028-1036.

29. Jackevicius CA, Paterson JM, Naglie G. Concordance between discharge prescriptions and insurance claims in post-myocardial infarction patients. Pharmacoepidemiol Drug Saf 2007; 16:207-215.

30. Gottlieb SS, McCarter RJ, Vogel RA. Effect of beta-blockade on mortality among high-risk and low-risk patients after myocardial infarction. $N$ Engl J Med 1998; 339:489-497.

31. Chandra A, Staiger D. Testing a Roy model with productivity spillovers: Evidence from the treatment of heart attacks. NBER Working Paper No. 10811, September 2004.

32. Stukel TA, Lucas FL, Wennberg DE. Long-term outcomes of regional variations in intensity of invasive vs medical management of Medicare Patients with acute myocardial infarction. JAMA 2005; 293:1329-1337.

33. Nobilio L, Ugolini C. Selective referrals in a 'hub and spoke' institutional setting: the case of coronary angioplasty procedures. Health Policy 2003; 63:95-107 


\section{LEGENDS}

Figure 1. Proportion of patients surviving hospitalization for acute myocardial infarction with post-hospitalization beta-blocker initiation, 2004-2007

Figure 2. Proportion of patients initiating beta-blocker therapy following hospitalization for acute myocardial infarction who were adherent, moderately adherent, and nonadherent within the first 6 months of treatment (Panel A) and within the first year of treatment (Panel B), 2004-2007 


\section{TABLES}

\section{Table 1. Selected demographics and characteristics of acute myocardial infarction hospitalization survivors in}

Regione Emilia-Romagna, 2004-2007*

All patients

hospitalized for

AMI $(n=24,367)$
Beta-blocker

initiators $(n=16,383)$
Beta-blocker noninitiators $(n=7,984)$

Age group, n (\%)

$<55$

$55-64$

$65-74$

$75-84$

$85+$

Male, n (\%)

Primary or secondary diagnoses in prior year, $\mathrm{n}(\%)$

Congestive heart failure

Angina pectoris

Other ischemic conditions

Other cardiovascular diseases

$\begin{array}{lll}3,135(12.9) & 2,347(14.3) & 788(9.9) \\ 4,334(17.8) & 3,303(20.2) & 1,041(13.0) \\ 6,019(24.7) & 4,370(26.7) & 1,649(20.7) \\ 7,394(30.3) & 4,716(28.8) & 2,678(33.5) \\ 3,475(14.3) & 1,647(10.1) & 1,828(22.9) \\ 15,584(64.0) & 10,845(66.2) & 4,739(59.4)\end{array}$

793 (3.2)

$384(2.3)$

409 (5.1)

$372(1.5)$

$262(1.6)$

$110(1.4)$

665 (2.7)

458 (2.9)

207 (2.6)

$2,447(10.0)$

$1,426(8.7)$

$1,021(12.8)$

Pre-hospitalization medications, n (\%)

Digoxin

ACE-inhibitors

Nitrates

Beta-blockers

$$
982(4.0)
$$

7,837 (32.2)

4,732 (19.4)

$5,710(23.4)$
$536(3.3)$

$5,348(32.6)$

3,062 (18.7)

$4,385(26.8)$
$446(5.6)$

2,489 (31.2)

$1,670(20.9)$

1,325 (16.6) 
$\mathrm{CCBs}$

ARBs

Secondary diagnoses with high mortality risk at index

admission, n (\%)

Cancer

Renal disease

Pneumonia

Cerebrovascular disease

Any one of high-risk secondary diagnoses

CCDG score in prior year, $\mathrm{n}(\%)$

$0-1$

2-3

$4+$

Index hospitalization length of stay, mean \pm SD

CABG during index hospitalization, $\mathrm{n}(\%)$

PTCA during index hospitalization, n (\%)

Cardiac catheterization during index hospitalization, n (\%)
$5,917(24.3)$

$3,636(14.9)$

$2,556(15.6)$

$4,036(24.6)$

$1,881(23.6)$

$1,080(13.5)$

$\begin{array}{ccc}1,354(5.6) & 797(4.9) & 557(7.0) \\ 827(3.4) & 363(2.2) & 464(5.8) \\ 3,611(14.8) & 1,974(12.0) & 1,637(20.5) \\ 15,980(65.6) & 10,424(63.6) & 5,556(69.6) \\ 16,439(67.5) & 10,703(65.3) & 5,736(71.8)\end{array}$

$\mathrm{SD}=$ standard deviation; $\mathrm{ACE}=$ angiotensin-converting enzyme; $\mathrm{CCBs}=$ calcium channel blockers; $\mathrm{ARBs}=$ angiotensin II $11,346(46.6) \quad 3,687(46.9) \quad 3,659(45.8)$

$9,804(40.2) \quad 6,628(40.5) \quad 3,176(39.8)$

$3,217(13.2) \quad 2,068(12.6) \quad 1,149(14.4)$

receptor blockers; $\mathrm{CCDG}=$ chronic condition drug groups; $\mathrm{CABG}=$ coronary artery bypass graft; $\mathrm{PTCA}=$ percutaneous transluminal coronary angioplasty

* See online appendix for full list of baseline demographics and patient characteristics 
Table 2. Selected independent predictors of beta-blocker initiation following acute myocardial infarction hospitalization discharge in Regione Emilia-Romagna, 2004-2007*

\begin{tabular}{lll}
\hline Variable & Odds Ratio & $95 \%$ Confidence Interval
\end{tabular}

Age group

$<55$

$55-64$

$65-74$

$75-84$

$85+$

Sex

Female

Male

Primary or secondary diagnoses in prior year

Congestive heart failure

0.87

1.27

1.26

0.95

Other cardiovascular diseases

Pre-hospitalization medications

Digoxin

0.97

$0.84-1.12$

ACE-inhibitors

1.19

$1.11-1.28$

Nitrates

1.00

$0.92-1.09$

Beta-blockers

CCBs
1.81

1.09
$0.87-1.09$

0.73-0.91

0.59-0.73

0.41-0.53
0.71-1.08

0.99-1.62

0.99-1.61

0.84-1.07 
ARBs

1.15

$1.05-1.26$

Secondary diagnoses with high mortality risk at index

admission

Cancer

$0.83-1.06$

Renal Disease

0.66

$0.56-0.76$

Pneumonia

0.88

$0.81-0.96$

Cerebrovascular disease

0.93

$0.76-1.15$

Any one of high-risk secondary diagnoses

1.06

$0.85-1.31$

CCDG score in prior year

$0-1$

$2-3$

$4+$

Index hospitalization length of stay (for every 5-day increase)

CABG during index hospitalization

PTCA during index hospitalization

Cardiac catheterization during index hospitalization
Referent

1.02

$0.94-1.11$

1.02

$0.91-1.15$

0.96

$0.95-0.97$
1.21

$1.11-1.32$

$\mathrm{SD}=$ standard deviation; $\mathrm{ACE}=$ angiotensin-converting enzyme; $\mathrm{CCBs}=$ calcium channel blockers; $\mathrm{ARBs}=$ angiotensin II receptor blockers; $\mathrm{CCDG}=$ chronic condition drug groups; $\mathrm{CABG}=$ coronary artery bypass graft; PTCA=percutaneous transluminal coronary angioplasty

* See online appendix for full list of predictors of beta-blocker initiation 